\title{
Super Pixel using Random Walk Segmentation
}

\author{
N.V. Andrews*, M. Manikandan and M. Paranthaman \\ Department of Electronics and Communication Engineering, \\ M. Kumarasamy College of Engineering (Autonomous), Karur - 639113, Tamil Nadu, India; \\ andrewsnv.ece@mkce.ac.in, manikandanm.ece@mkce.ac.in, paranthamanm.ece@mkce.ac.in
}

\begin{abstract}
Objectives: An innovative image superpixel segmentation approach run on the test image to acquire the probabilities of every pixel and performs grouping in light of shading similarities and spatial nearness of the pixels in histopathologic images. LRW algorithm using self-circles has the benefits of portioning the weak limits and confounded surface areas extremely fit. Method of analysis: This technique starts with instating the seed locations and tracks the LRW algorithm on the test image to acquire the probabilities of every pixel. At that point, the limits of starting superpixels is acquired by the probabilities what's more, the drive time. Findings: At the point when the outcomes are assessed, it has been watched that the superpixel strategy has a positive commitment to both the segmentation achievement and the running time. The execution of superpixel is enhanced using moving the focus places of super pixels then separating the huge superpixels into little with the proposed improvement procedure. The exploratory outcomes have shown that our technique accomplishes preferable execution over past superpixel approaches. Application/Improvements: To detect the distance objects as a test image, and through this method, we can analyze and provide an optimal solution where time is constraint.
\end{abstract}

Keywords: Features Classification Forest, Grey Level Co-Occurrence Matrix, Single Value Decomposition

\section{Introduction}

Superpixels are normally known as narrowing and gathering unbroken pixels in the picture, which is generally utilized as a part of numerous PC vision applications, for example, picture division and question acknowledgment. The superpixel idea was initially displayed as knowing the uniform districts utilizing the standardized cuts (NCuts) calculation. Primary value of superpixel is to give a added characteristic and significant portrayal of the info picture. In this manner, contrasted with the conventional pixel portrayal of the picture, the superpixel portrayal incredibly lessens the quantity of picture natives and enhances the agent efficiency $\mathrm{y}^{1,2}$.

Moreover, it will be helpful also viable to process the district established pictorial highlights by superpixels, which will give the essential benefits to the vision errands, for example, Protest acknowledgment. Here is a vast volume of writing on programmed superpixel calculations, for instance, standardized cuts, mean move calculation, chart based technique, Turbo pixels, SLIC superpixels and enhancement based superpixels. In any case, each superpixel technique has its own particular favorable position and disadvantage that might be more qualified for a specific application. It is as yet difficult to build up a top notch superpixel calculation, which keeps away from the under-partition and locally bunches the pixels regarding the power limits. The coveted possessions of a perfect superpixel calculation not just holds fast well to protest limits of picture, yet additionally keep up the reduced obliges in the convoluted surface districts.

${ }^{*}$ Author for correspondence 


\section{Supplementary Works}

A lot of papers have proposed different picture superpixel techniques amid the most recent years; concisely survey these whole things in this segment. The current superpixel methodologies will be generally categorized into two classifications. The major class is the calculations that don't reflect the smallness compels amid the superpixels age methodology, for example, mean move, and chart based calculations. To evade the superpixels crossing the protest limits, these division calculations by and large deliver the superpixels by finished portioning the picture. Since these calculations don't consider the smallness obliges, which may deliver the superpixels of very sporadic figures and dimensions. The next classification of superpixel calculations considers the minimization compels, for example, standardized cuts, cross section cut, Turbo Pixels, and diagram cut approaches ${ }^{3,4}$.

The objective of superpixel is to over-portion the info picture into little smaller districts thru standardized look. The superpixel division can be assumed as a pixel marking issue where every superpixel is doled out to an exceptional name. In this method starts with putting the instated seeds of the allocated superpixels. At that point, we utilize the LRW calculation to acquire the underlying superpixels and their limits. Keeping in mind the end goal to additionally variety the superpixels added conservative and their limits more predictable with the protest limits in picture, we build up a different vitality improvement calculation to streamline the seed locations and fragmented the extensive superpixels. New approach introduced an efficient Turbo Pixel superpixel calculation utilizing the level set based regular flow advancement from the consistently set seeds in the picture. Be that as it may, it showed moderately poor limit adherence in light of its numerical solidness issues particularly with convoluted Surfaces. Author built up a picture superpixel method through the diagram cut streamlining, in addition the superpixels acquired by sewing each pixel that had a place with just a single of the covering picture areas. Around other critical superpixel methods were proposed to accomplish the need of expanding applications. Moore et al. utilized a solitary chart slice technique to build an ideal arrangement in whichever the even or vertical heading, considered both the edges and the lucidness of coming about superpixel cross sections ${ }^{5,6}$.
Proposed a cross section similar arrangement of superpixel locales by uniform dimension by taking in the Eigen-pictures from the info picture, which enhanced the advancement quickness in the Turbo Pixel system. They exhibited a basic straight iterative grouping (SLIC) superpixel calculation, and embraced the k-implies bunching way to deal with produce the superpixels with moderately bring down computational cost.

The superpixel division issue as a target works scheduled the entropy rate in the diagram. The entropy rate will group the reduced and homogeneous areas, which likewise support the superpixels to cover with a solitary question, arranged the perceptual limits. The RW calculation created by Grady is an extremely famous and driving methodology for intelligent picture division utilizing the closer view and foundation seeds by the client.

\section{Proposed Work}

With a specific end goal to fulfill these coveted necessities, we build up another picture superpixel division technique by the apathetic irregular walk (LRW) and vitality improvement calculation to accomplish preferred execution over the past methodologies. Our picture superpixel division calculation depends on the summed up irregular walk (RW) calculations. Be that as it may, the first RW calculation relies upon the nearby connection among the pixel and its equivalent seeds by the first coming probability.

It prompts the unequal form of the final non-even superpixel comes about. Through seeing the worldwide connections among every one of the pixels and the seed focuses, we at that point build up an original superpixel calculation utilizing the LRW with the conservativeness limitations. Our LRW calculation with self-circles successfully takes care of the division issue in powerless limit and complex surface locales. Then again, the LRW based superpixel calculation may experience the ill effects of the affectability of the underlying seed positions. Keeping in mind the end goal to beat these restrictions and enhance the execution, we additionally build up another superpixel advancement approach by introducing a vitality streamlining system. Our superpixel advancement methodology is basically a conservativeness limitation, which guarantees the subsequent superpixels to convey 
consistently with the homogeneous size by migration and part component. Our vitality work is made dual things, the first information thing adaptively improves the places of seed focuses to brand the superpixel limits cling to the question limits thriving, and the additional smooth thing adaptively isolates the huge superpixels into little ones to variety the superpixels more standardized. As per these migrated seed positions and recently made seeds by the part conspire, our LRW calculation is performed again to enhance the underlying superpixels, which brands the limits of ending superpixels hold fast to protest
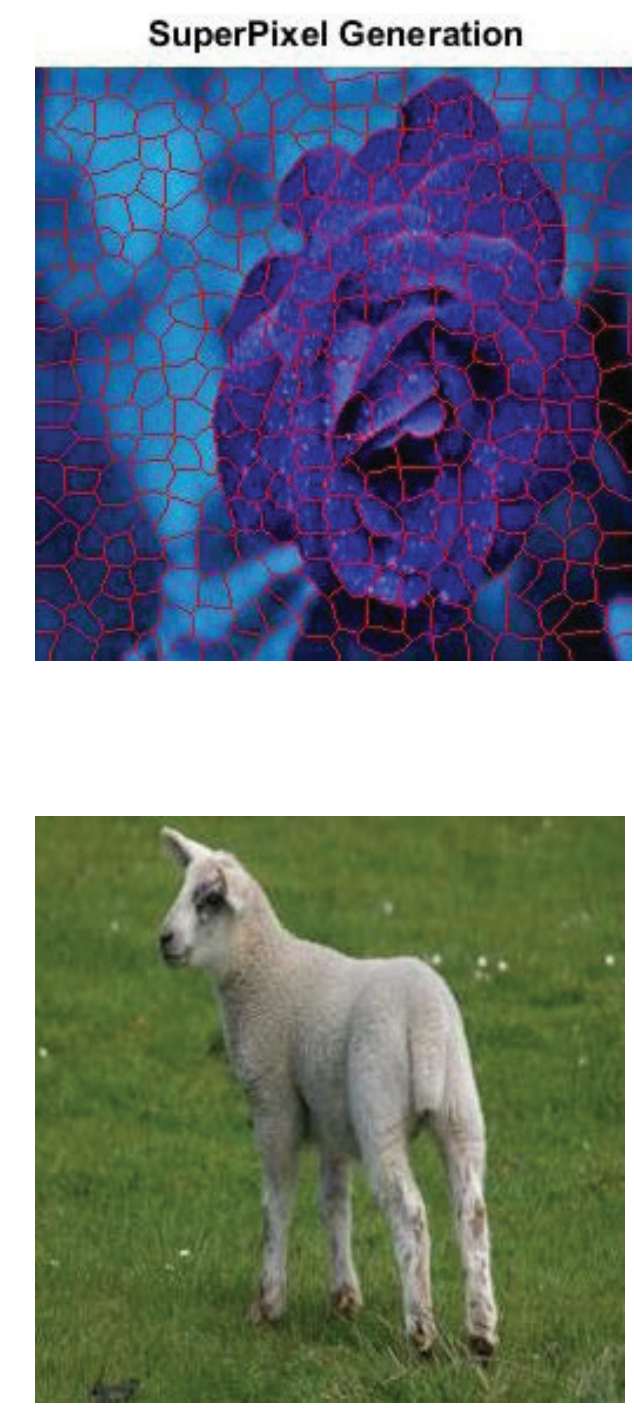

Figure 1. Input and superpixel. limits extremely fine. Our basis program and additional resources will be openly accessible on the web ${ }^{7-9}$.

Figure 1 shows the input and corresponding superpixel identification for the given input. As far as semi-managed grouping, a class of name spread calculations through irregular stroll on diagram emerges from the group. The irregular walk demonstrate was right off the bat connected in Web field, for example, PageRank, that is a fruitful occurrence of such model, given a model engrossing states, which can be utilized for content rundown and informal organization investigation. Then again, the

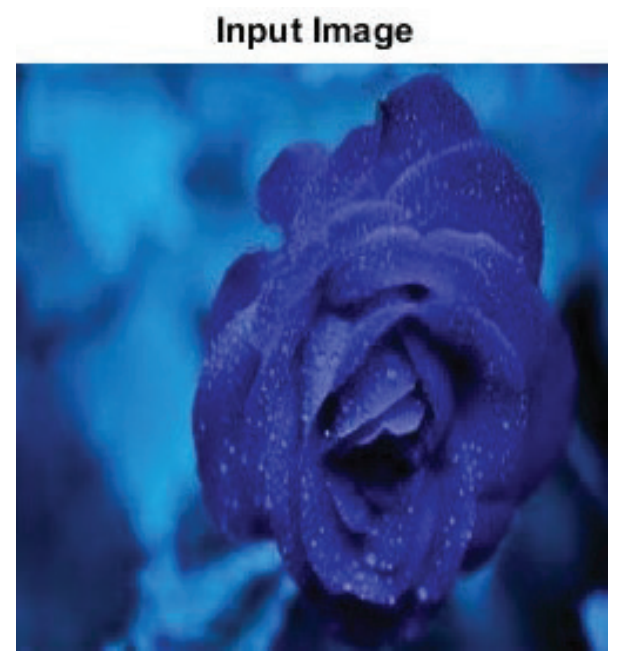

\section{SuperPixel Generation}

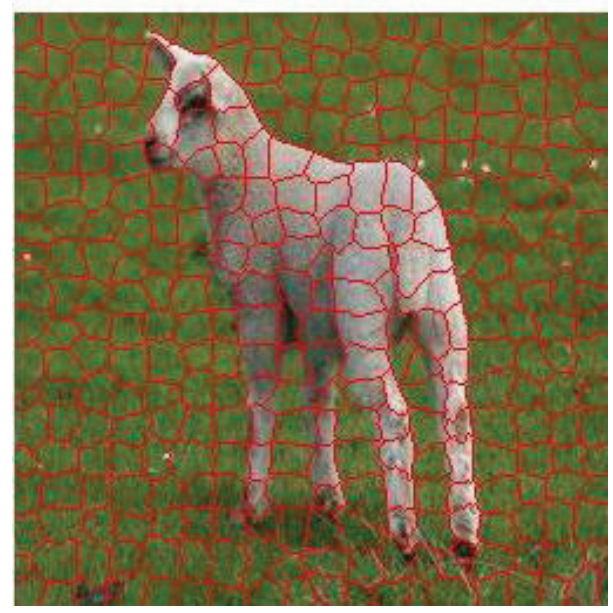


arbitrary stroll on chart is progressively bunching and arrangement issues. As to name proliferation thoughts, as a rule, they take care of the grouping issue through spreading the marks of named tests to adjacent unlabeled examples in view of the bunch and complex suspicion. Despite the fact that the name spread thoughts are natural, and has made certain accomplishments, however the mix of some unlabeled information in semi-regulated learning isn't continually working, which is known to all. On the off chance that the earlier information isn't completely solid because of the nearness of clamor or beguiling, at that point the semi-regulated strategy won't just bound to build the measure of related activities, yet even deliver more noteworthy commotion, along these lines influencing the characterization comes about.

Taking the biomedical information as case, because of the unavoidable mistakes amid their accumulation, these informational indexes will fundamentally contain some boisterous examples, in this manner how to expand the lessening of clamor impedance is a major issue. In perspective of the above issues, we propose a managed classifier apathetic irregular stroll based on our previous business related to arbitrary walk learning on diagram. We begin by presenting a lethargic arbitrary walk demonstrates as base classifier, at that point from the successful utilization of named information; we introduce an enhanced classifier through the complementally combinatorial improvement. The grouping execution is tried on various bio-therapeutic informational indexes and examinations with other administered classifiers are led, comes about demonstrated that our exhibited classifier in this paper can enhance the execution and adequacy of information order. Given a game plan of midway named tests, the general issue of request or desire unites the taking in process from the given data so as to allot names to those unlabeled illustrations.

\section{Expected Result}

Likewise, customary information mining approaches frequently require solid earlier presumptions or adequate preparing tests, consequently influencing their scope of utilization.

For example, in page order, couple of online client's wills compensation much time for preference their intrigued website pages as the marked occurrences; in the meantime, there are a huge number of site pages going about as the unlabeled cases. Along these lines, how to make full utilization of the restricted earlier information

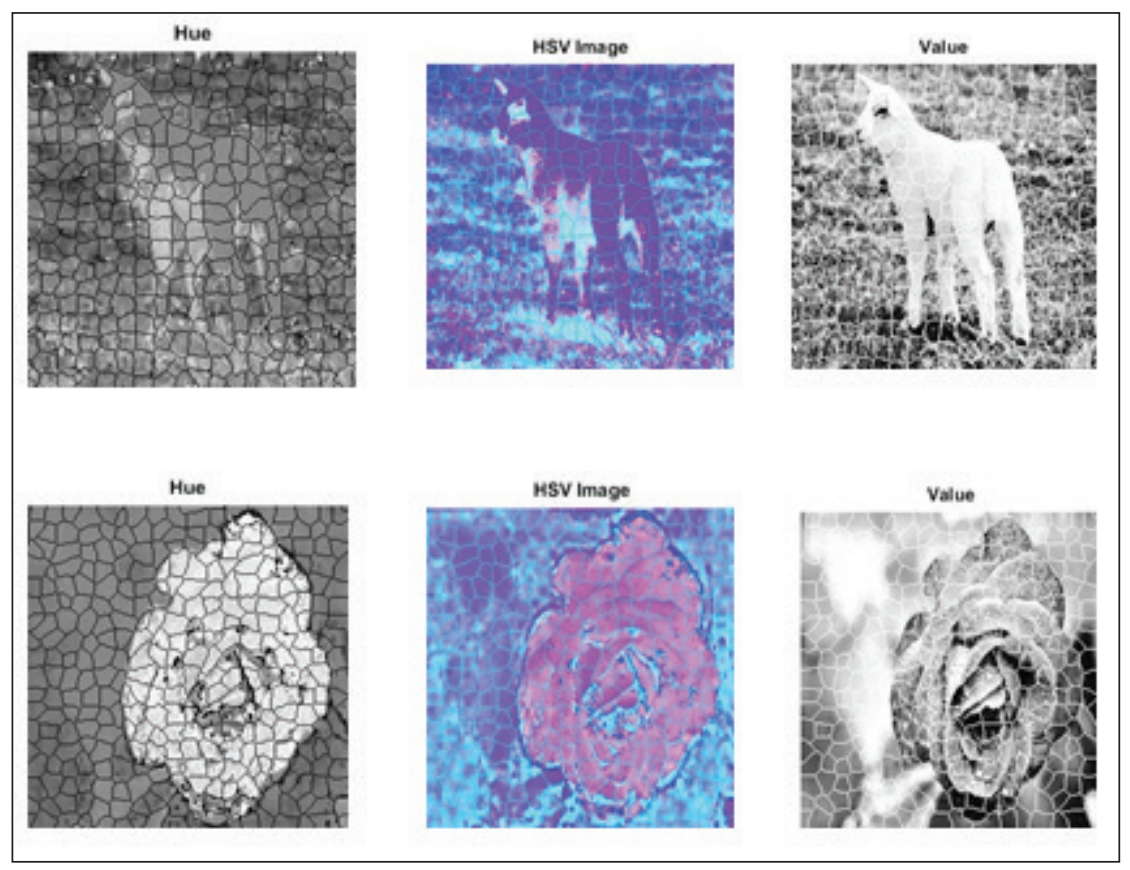

Figure 2. Hue, HSV and Value of the input images. 
turns into an issue deserving of study. In Figure 2 the RW calculation accomplishes the preferred intuitive picture division execution over the diagram cut based division technique with a similar client association.

Grady and Schwartz additionally displayed an original isoperimetric diagram apportioning method with a little isoperimetric consistent and accomplished fantastic divisions by understanding a straight framework, which was a powerful seedless rendition of the RW calculation. Additionally, broadened the RW calculation by in corporating a non-parametric probability density model towards find detached items for division. However, in Figure 3 the RW calculation for intuitive picture division does not have a worldwide shading dispersion demonstrate, which will influence the division to come about touchy to the locations and amounts of the client defined seeds on the picture objects. In order to give cannier approaches to comprehend the expectation of client image, proposed an obliged RW calculation to encourage the different client contributions with an intuitive altering structure. The technique made exact shape refinement of the division comes about. Built up a power watershed division system, controlled the RW, briefest way streamlining and diagram cuts.
Administered learning includes two phases for grouping procedure, first is the knowledge (exercise) arrange, in Figure 4 a classifier is worked by examining or gaining from the preparation information. Blend enhancement is a typical method to fabricate a phenomenal grouping perfect; here we attempt to utilize lethargic walk classifier as corrupt student for prepare an extra powerful classifier over the combinatorial enhancement. Before proposing the combinatorial enhancement technique, we initially present the damage of grouping; it is a critical metric for grouping execution.

\section{Discussion}

The departure of a solitary classifier in ordering the whole informational collection is created of the difficult misfortune and preparing misfortune. For the most part, testing misfortune has taken to think about the execution of a classifier. We as a whole know keeping in mind the end goal to prepare a viable classifier, the named information ought to be completely used; in the meantime, the impact of clamor inside the preparation information ought to be stayed away from, particularly when the informational

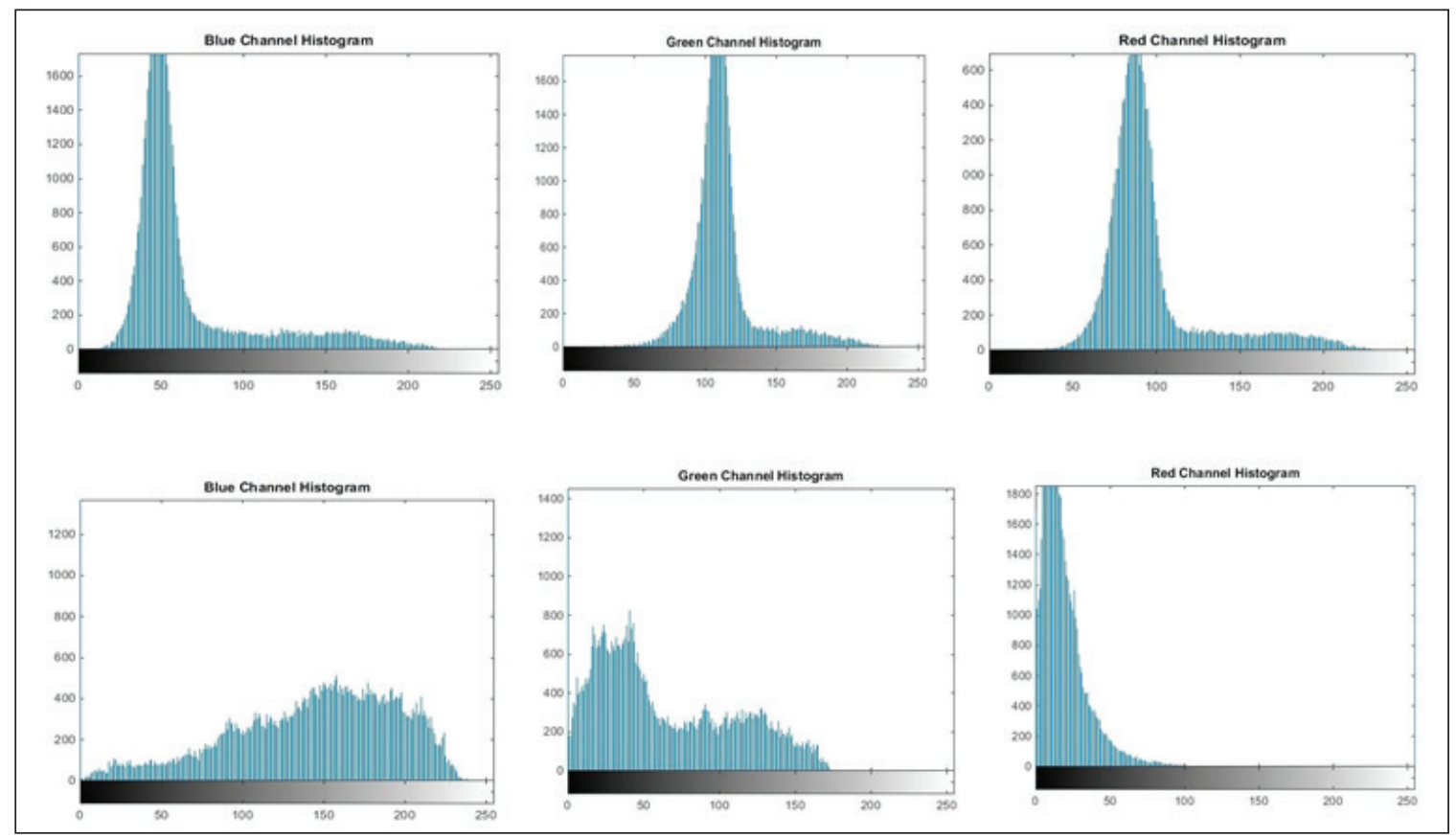

Figure 3. Histogram of RGB. 


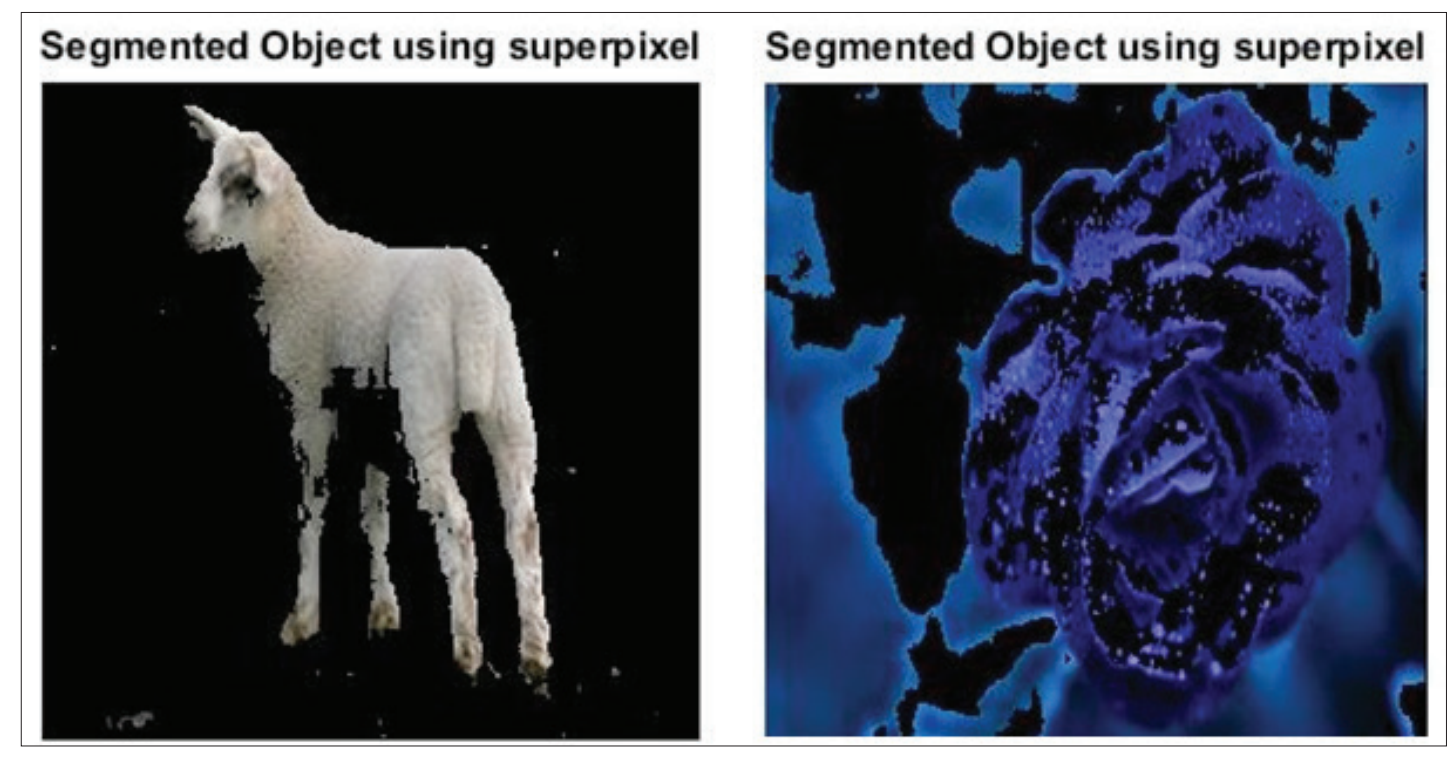

Figure 4. Expected output.

index to be arranged contains a specific measure of commotion. This first entry likelihood disregards the entire connection among the present pixel and different kernels.

As meant by, Restrictions of the first RW technique provide the purpose that it experiences the powerless limit and difficult surface divisions. Keeping in mind the end goal toward kind full utilization of the worldwide connection among the pixel and every one of the seeds, we include the self-circle above the diagram top to kind the RW procedure lethargic, which is enlivened via the first LRW idea. Be that as it may, the first LRW was at first proposed for the site information grouping and mining applications. In this application, need to have to additionally build up the first LRW calculation to be reasonable for our picture superpixel division application.

As appeared in Figure 3, the fundamental commitment of LRW grounded superpixel calculation is two-overlap. But, the self-circle is included every vertex to guarantee the limit compel for superpixels. Since a vertex with an overwhelming self-circle will probably assimilate its adjacent pixels than the one through light self-circle, which types the vertex to ingest and catch both the feeble limit and surface data with self-circles. Then again, rather than beginning after the pixels to the kernel focuses as the first
RW calculation. Here segment, assess the planned LRW calculation. The superpixel comes about are examined to clarify the influence through various limit surroundings. The future superpixel calculation is subjectively tried with a few agent cases in BSD benchmark. All together exhibited a novel picture superpixel approach utilizing the LRW and vitality streamlining calculation. Here technique first runs the LRW calculation to get the underlying superpixel results.

\section{Conclusion}

The exhibited technique is tried on ten informational collections including parallel and different grouping issues in correlation to three seat strategies. Our calculation absolutely beats them by a limited preferred standpoint. All things considered, our proposed classifier isn't just instinctive and successful, yet additionally appears amazing arrangement execution. Going for arrangement issue, a great classifier will continuously investigate and abuse the important data out of marked information, and will likewise be strong for clamor, with the goal that it can lessen the preparation misfortune to a substantial degree and increments the forecast precision. 


\section{References}

1. Bai X, Sapiro G. A geodesic framework for fast interactive image and video segmentation and matting. IEEE 11th International Conference on Computer Vision; 2007. p. 1-8. Crossref.

2. Moore A, Prince S, Warrel J. Lattice cut-Constructing superpixels using layer constraints. IEEE Computer Society Conference on Computer Vision and Pattern Recognition; 2010. p. 2117-24.

3. Yang W, Cai J, Zheng J, Luo J. User-friendly interactive image segmentation through unified combinatorial user inputs. IEEE Transactions on Image Processing. 2010; 19(9):2470-9. Crossref. PMid:20716497

4. Gopalakrishnan V, Hu Y, Rajan D. Random walks on graphs for salient object detection in images. IEEE Transactions on Image Processing. 2010; 19(12):3232-42. Crossref. PMid:21078566
5. Couprie C, Grady L, Najman L, Talbot H. Power watershed: A unifying graph-based optimization framework. IEEE Transactions on Pattern Analysis and Machine Intelligence. 2011; 33(7):1384-99. Crossref. PMid:21079274

6. Pätz T, Preusser T. Segmentation of stochastic images with a stochastic random walker method. IEEE Transactions on Image Processing. 2012; 21(5):2424-33. Crossref. PMid:22334006

7. Wang J, Wang X. VCells: Simple and efficient superpixels using edge-weighted centroidal Voronoi tessellations. IEEE Transactions on Pattern Analysis and Machine Intelligence. 2012; 34(6);1241-7. Crossref. PMid:22331852

8. Sheikdavood K, Mahamudha P, Nagendran K. Performance evaluation on accurate coronary centerline extraction and catheter detection in angiographies. International Journal of Applied Engineering Research. 2015; 10(1):349-53.

9. Rajan SP, Davood SK. Performance evaluation on automatic follicles detection in the ovary. International Journal of Applied Engineering Research. 2015; 10(55):1-5. 